los herbívoros invasores (erradicación de arruí y muflón y mayor control del conejo en las zonas aledañas a las poblaciones actuales). Además, al menos para $V$. guaxarensis los modelos reflejan la necesidad de favorecer en el futuro su migración de forma asistida, haciendo traslocaciones al área que podemos considerar como refugio climático en el estratovolcán Teide-Pico Viejo, siempre y cuando se eviten posibles problemas de hibridación con V. cheiranthifolia.

\begin{tabular}{|c|c|c|c|c|}
\hline & $\begin{array}{c}\text { Pasado } \\
1959-1989\end{array}$ & $\begin{array}{l}\text { Presente } \\
1990-2019\end{array}$ & $\begin{array}{c}\text { Futuro RCP } 8.5 \\
2041-2060\end{array}$ & $\begin{array}{c}\text { Futuro RCP } 8.5 \\
2061-2080\end{array}$ \\
\hline \multicolumn{5}{|l|}{ Superficie (ha) } \\
\hline V. palmensis & 2.988 & 1.784 & 648 & 148 \\
\hline V. cheiranthifolia & 7.022 & 3.745 & 2.332 & 1.860 \\
\hline V. guaxarensis & 7.960 & 6.152 & 4.148 & 3.132 \\
\hline \multicolumn{5}{|c|}{ Velocidad media de respuesta al cambio climático (m / año) } \\
\hline V. palmensis & 21,1 & - & 15,7 & 20,0 \\
\hline V. cheiranthifolia & 19,6 & - & 54,0 & 30,2 \\
\hline V. guaxarensis & 11,0 & - & 21,2 & 25,1 \\
\hline
\end{tabular}

Tabla 1. Datos de superficie y velocidad media de respuesta al cambio climático de Viola spp. en los diferentes escenarios.

\section{Bibliografía}

- Cubas J., Irl S. D., Villafuerte R., Bello-Rodríguez V., Rodríguez-Luengo J. L., Del Arco M., Martín Esquivel J.L. \& J.M. González-Mancebo (2019) Endemic plant species are more palatable to introduced herbivores than non-endemics. Proceedings of the Royal Society B, 286(1900), 20190136.

- Del Arco M.J. (2008) La flora y la vegetación canaria ante el cambio climático actual. Naturaleza amenazada por los cambios en el clima Actas III Semana Científica Telesforo Bravo, pp, Tenerife: Instituto de Estudios Hispánicos de Canarias, 105-140.

- Gobierno de Canarias. Banco de Datos de Biodiversidad de Canarias (http//:www.biodiversidadcanarias.es(biota) [ Julio de 2021].

- Hamann A., Roberts D. R., Barber Q. E., Carroll C. \& S.E. Nielsen (2015) Velocity of climate change algorithms for guiding conservation and management. Global Change Biology 21(2): 997-1004.
- Karger D.N., Conrad O., Böhner J., Kawohl T., Kreft, H. Soria-Auza R.W.' Zimmermann N.E. Linde P. \& M. Kessler (2017) Climatologies at high resolution for the Earth land surface areas. Scientific Data. 4: 170122.

- Marrero-Gómez M. V., Martín Esquivel J. L., Docoito Díaz J. R. D. \& M.S. Izquierdo (2020) Viola guaxarensis (Violaceae): a new Viola from Tenerife Canary Islands, Spain. Willdenowia 50(1): 13-21.

- Martín J L Bethencourt J \& E Cuevas-Agulló (2012) Assessment of global warming on the is land of Tenerife, Canary Islands (Spain). Trends in minimum, maximum and mean temperatures since 1944. Climatic Change 114(2): 343-355.

- Phillips S. J., Anderson R. P. \& R.E. Schapire (2006) Maximum entropy modeling of species geographic distributions. Ecological modelling 190(3-4): 231 259.
- Rodríguez-Rodríguez P. G. Fernández de Castro A Seguí J., Traveset A., \& P.A. Sosa (2019) Alpine species in dynamic insular ecosystems through time: conservation genetics and niche shift estimates of the endemic and vulnerable Viola cheiranthifolia. Annals of Botany 123(3): 505-519.

- Seguí J., López-Darias M., Pérez A.J., Nogales M \& A. Traveset (2017) Species-environment interactions changed by introduced herbivores in an oceanic high-mountain ecosystem. AoB Plants 9 : plw091.

VÍCTOR BELLO-RODRÍGUEZ1', JONAY CUBAS', MARCELINO J. DEL ARCO', JOSÉ L. MARTÍN ESQUIVEL², MANUEL V. MARRERO-GÓMEZ², JUANA MARÍA GONZÁLEZ-MANCEBO

- Grupo de investigación Plant Conservation and Biogeography. Departamento de Botánica, Ecología y Fisiología Vegetal, Universidad de La Laguna. Avda. Francisco Sánchez s/n, 38206 La Laguna, S.C. de Tenerife, España.

2. Parque Nacional del Teide, C/. Dr. Sixto Perera González, 25, 38300 La Orotava, Tenerife, Islas Canarias, España

\title{
El censo como herramienta para conocer y proteger La Tejeda del Mosquito do: 10155660202125004
}

\section{The census as a tool to know and protect La Tejeda del Mosquito}

\begin{abstract}
Resumen / Abstract
Unos kilómetros al norte del Hayedo de Montejo, en la zona conocida como El Mosquito, la Asociación de Amigos del Tejo y las Tejedas (AATT) ha realizado un censo de Taxus baccata L. en el que se han contabilizado 8.304 ejemplares. Alrededor de la mitad (54\%) tiene entre $5 \mathrm{~cm}$ y $1,5 \mathrm{~m}$ de altura mientras el resto presenta mayor talla, incluso hasta perímetros de tronco superiores a $3 \mathrm{~m}$. La mayor parte se localizan en zonas muy concretas, ocupando el grueso de la población unas 55 ha, y con una distribución estructural y espacial que demuestra la plasticidad de la especie. El tamaño y la densidad de esta población supera la de cualquier otra de la Comunidad de Madrid y del Sistema Central conocidas hasta el momento, y se ha determinado que el área estudiada tiene todas las características exigidas para ser considerada Hábitat 9580*: Bosques mediterráneos de Taxus baccata L., con las peculiaridades en la composición de las especies acompañantes inherentes a los bosques mediterráneos, acentuadas por su influencia atlántica.
\end{abstract}

A few kilometers north of Hayedo de Montejo, in an area called El Mosquito, the Spanish Association of Friends of the Yew has carried out a census of Taxus baccata L., that led to counting 8.304 specimens ranging from $5 \mathrm{~cm}$ to $1,5 \mathrm{~m} \mathrm{high} \mathrm{(54 \%} \mathrm{of} \mathrm{them)} \mathrm{to} \mathrm{above} 3 \mathrm{~m}$ of perimeter at breast height. Most of them are located in very specific clusters with the bulk of the population in about 55 ha and with a spatial and structural distribution that demonstrates the plasticity of the species. The size of the population exceeds any other yew grove known in the Community of Madrid and the whole Central System. The study area has all the requirements to be classified as Habitat 9580*: Mediterranean forests of Taxus baccata L. with the peculiarities in the composition of accompanying species inherent to Mediterranean forests, accentuated by its Atlantic influence.

Palabras clave / Keywords

Tejeda, regeneración, censo, hábitat 9580*

Yew grove, rewilding woodland, census, habitat 9580* 


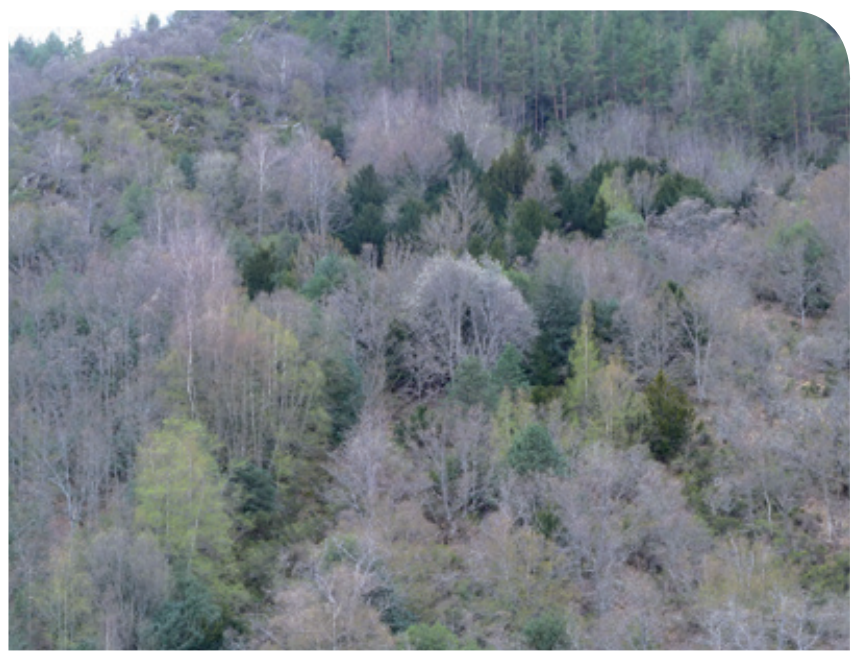

Figura 1. Ladera de El Mosquito en la que se observa un núcleo de tejos inmerso en el bosque mixto caducifolio.

\section{Introducción}

En la cabecera del río Jarama se localiza una notable población de tejos, en la que recientemente se ha realizado un detallado estudio con un censo muy preciso del total de ejemplares (AATT, 2021). En esta zona, los aprovechamientos tradicionales desde la Edad Media han sido ganaderos (ganado ovino, caprino y vacuno), acopio de leña, carboneo y el cultivo de centeno. Además, se mantenían algunos pies centenarios de diversas especies arbóreas de forma adehesada, que eran utilizados como lugar de sesteo y sombra para los rebaños en verano.

Durante la Guerra Civil y en la posguerra casi desaparece el ganado vacuno y se incrementa el cultivo de centeno. Hacia 1960 el Estado compra y expropia el MUP 156 "Sierra Escalva", prohíbe su utilización para uso ganadero y planta pinos en cotas intermedias, donde se corta la mayoría de los grandes robles. Gran parte del ganado ovino y caprino desaparece y los pinares se dejan crecer sin cortas selectivas a los 20-30 años de la plantación. La regeneración de la vegetación natural comenzó en las cercanías de los cauces de agua y en las orlas del pinar, donde la presencia de roquedos impidió su establecimiento y, progresivamente, ocupó las terrazas inferiores. Una de las especies que incrementó notablemente su presencia en la zona fue el tejo (Taxus baccata L.), comportándose con extraordinaria pujanza como especie colonizadora y climácica, y motivando un gran interés para conocer la densidad, estructura y dinámica de sus poblaciones.

\section{Material y métodos}

La zona conocida como El Mosquito se localiza en la margen derecha de la cabecera del río Jarama, en umbría, al noreste de la Comunidad de Madrid, en el entorno Somosierra-Sierra de Ayllón, en el Sistema Central de la Península Ibérica. El área de estudio comprende desde las fuentes del río Jarama en el paraje de Reajos Llanos (1.900 m s.n.m.) hasta el arroyo de la Caseta (1.370 m s.n.m.), con una superficie aproximada de 265 ha. También se ha incluido en el estudio el Monte del Matazo, en la margen izquierda de la cabecera del río Jarama, que se localiza en solana y en la Comunidad de Castilla-La Mancha. En el área el sustrato está constituido por rocas metamórficas (paraneises, esquistos) de edad Varisca y composición fundamentalmente ácida, mientras que la cobertura cuaternaria es escasa (IGME, 1991, 2005). Los suelos más abundantes son suelos pardos ácidos, presentes bajo cubierta forestal, y rankers de pendiente (Perea, 2011).

La cabecera del río Jarama se encuentra en la región biogeográfica mediterránea, provincia Mediterránea-lbérica-Occidental, sector Guadarrámico, subsector Ayllonense. El ambiente es submediterráneo templado oceánico y el termoclima pertenece al piso supra-submediterráneo (Rivas Martínez, 1987), las sequías estivales son cortas y los periodos vegetativos breves al alargarse los meses fríos. Las elevaciones de los cordales de la Sierra fomentan una mayor precipitación que en otros sectores del Sistema Central y la mayor recurrencia de tormentas estivales en el tramo oriental de Guadarrama-Ayllón favorece el arbolado eurosiberiano con Quercus petraea, Betula pubescens, Fraxinus excelsior, etc., dentro del robledal de Q. pyrenaica (Fig. 1).

Entre abril de 2017 y enero de 2020 se han realizado un total de 21 jornadas de censo y otras 11 de reconocimiento. En el trabajo de campo participaron de manera voluntaria y altruista cuadrillas formadas por 3 o 4 personas, que recorrían el terreno en paralelo barriendo zonas contiguas. Se contabilizaron todos los ejemplares mediante visualización directa, anotando coordenadas y altitud por GPS, tipo, altura, perímetro, afecciones, sexo y ecología y se realizó una estimación del catálogo florístico del área de estudio. La tipología de cada ejemplar se clasificó de acuerdo a Fernández González et al. (2015) y Balaguer Romano et al. (2020) en:

- Tipo 1. Regenerado: ejemplares entre $5 \mathrm{~cm}$ y 1,5 m de altura.

- Tipo 2. Juvenil: ejemplares de más de 1,5 m de altura y menos de $50 \mathrm{~cm}$ de perímetro a altura de pecho.

- Tipo 3. Adulto: ejemplares de más de 1,5 m de altura y perímetro entre 50 y $300 \mathrm{~cm}$.

-Tipo 4. Viejo: ejemplares de perímetro superior a $300 \mathrm{~cm}$.

En el análisis y representación cartográfica se ha utilizado la cartografía del Instituto Geográfico Nacional y de la Comunidad de Madrid y las ortofotos del Plan Nacional de Ortofotografía Aérea (PNOA) de 2007 a 2018; los fotogramas de los vuelos americanos, series A (1945-46) y B (1956-57), y los fotogramas de los vuelos interministeriales de los años 1973 a 1986. La distribución espacial se analizó mediante el programa QGIS 3.10.

Se ha utilizado el índice de rejuvenecimiento (Ir), entendido como la suma de individuos de regeneración más juveniles dividido por el número total de individuos (Balaguer Romano et al., 2020). Además, se ha completado la información sobre el área de estudio con referencias bibliográficas y con comunicaciones personales sobre datos y topónimos no referenciados de agentes medioambientales, botánicos, y vecinos de la zona.

\section{Resultados y discusión}

En las 265 ha prospectadas se censaron un total de 8.304 tejos. 4.472 ejemplares pertenecen al tipo 1 (54\%), 3.007 al tipo 2 (36 \%), 820 al tipo $3(9,9 \%)$ y 5 al tipo 4 (0,1\%). El grueso de la población se concentra en una superficie de 55 ha, con una densidad media de 149 pies/ha, llegando a alcanzar una densidad máxima de 2.550 pies/ha (Fig. 2).

En el total del área poblacional se reconocen cinco núcleos principales o subpoblaciones que tienden a agruparse en vaguadas. Los lomos, más rocosos, venteados y secos, interrumpen la continuidad de estas subpoblaciones. 


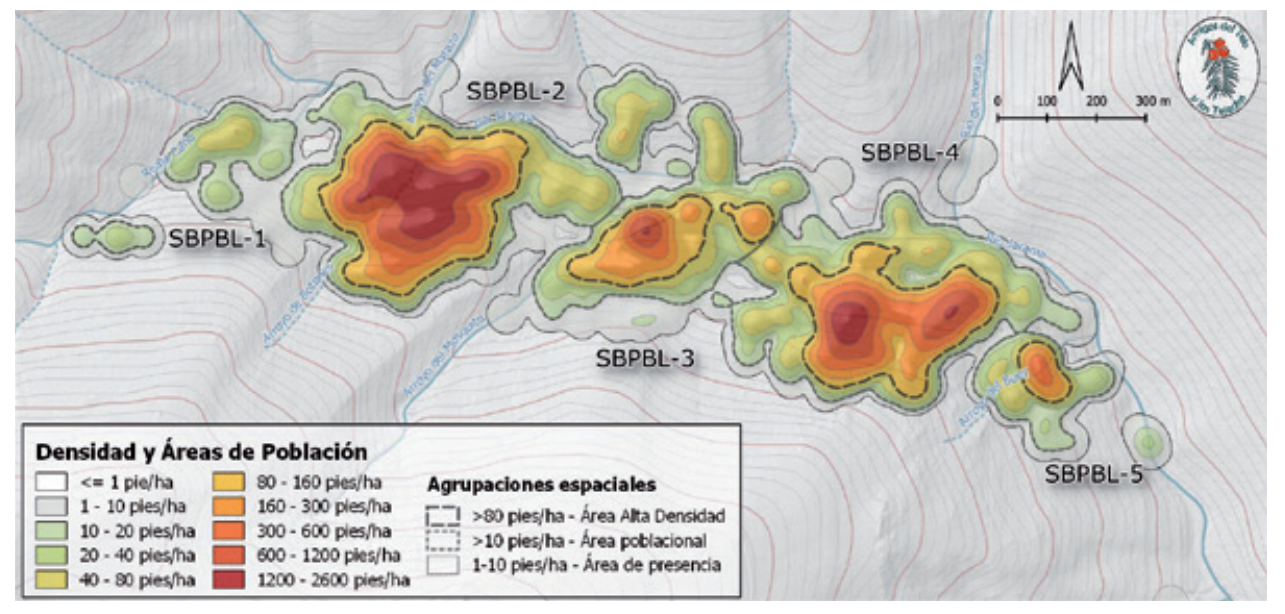

Figura 2. Variación en la densidad de Taxus baccata y agrupaciones espaciales (AATT, 2021).

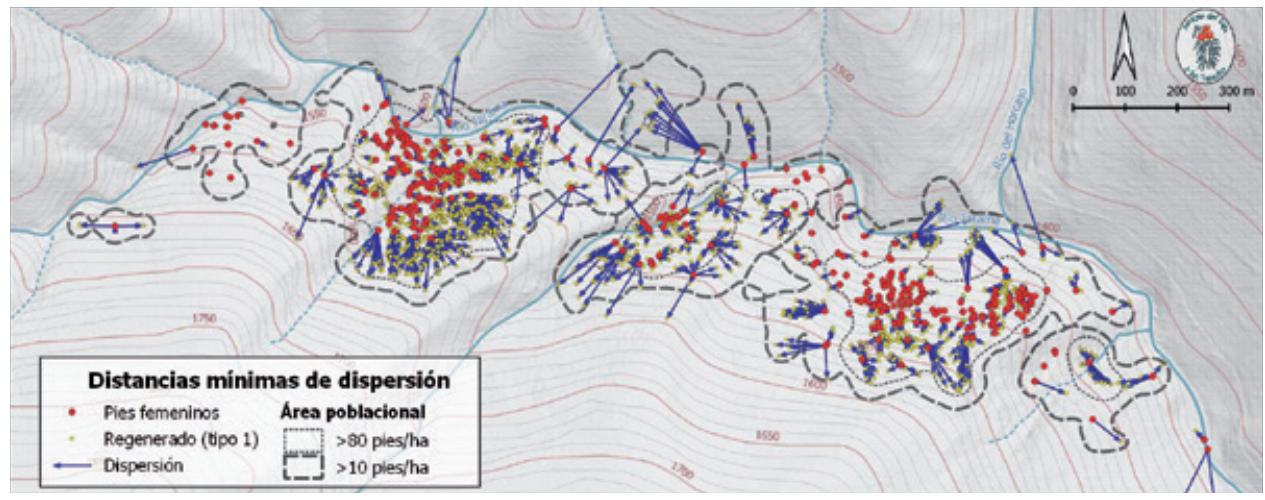

Figura 3. Áreas poblacionales y distancias mínimas de dispersión de las semillas (AATT, 2021).

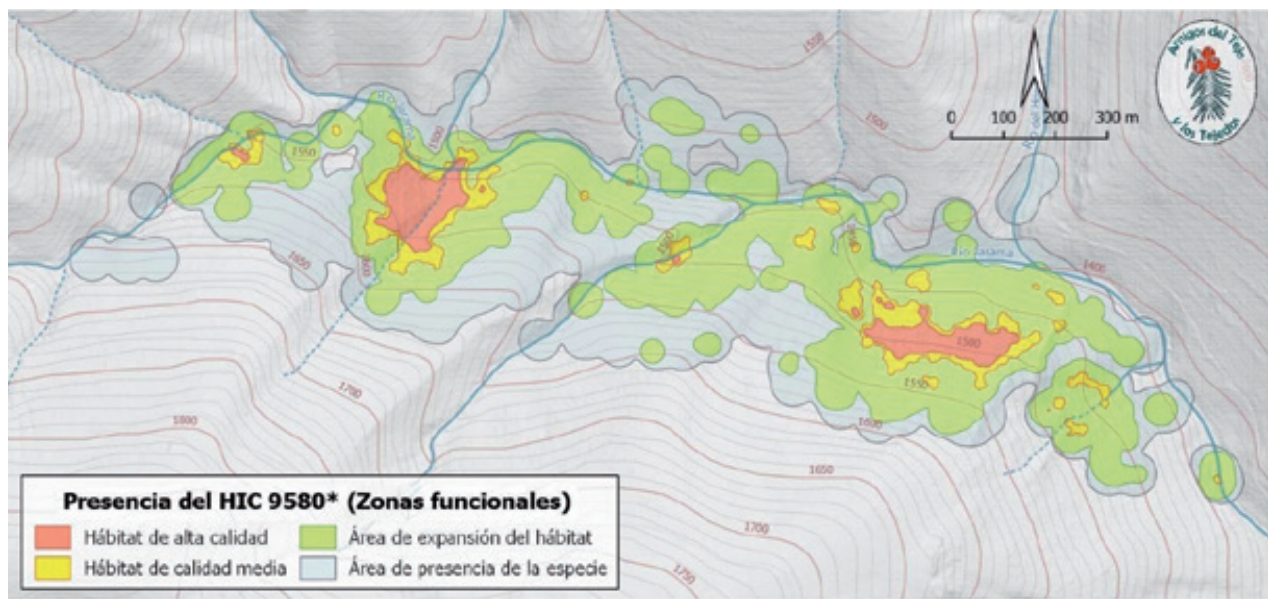

Figura 4. Zonificación del territorio estudiado en función de la calidad del Hábitat 9580

En algo más de un tercio de la extensión total, la formación de tejos se encuentra bien establecida con individuos del tipo 3 o 4, y casi en el $90 \%$ de la extensión se encuentran individuos del tipo 1 o 2, lo cual evidencia una regeneración pujante.

Las condiciones ambientales y microclimáticas difieren entre las distintas subpoblaciones: en las subpoblaciones 1, 2 y 3, bajo pinar, el tejo tiene altas tasas reproductivas, mientras que en 4 y 5, bajo caducifolios, las subpoblaciones están más asentadas y presentan una estructura más evolucionada. Además, se ha determinado una predominancia de hembras sin apreciable desviación entre individuos adultos y juveniles, relación que en general se considera como óptima.

El valor obtenido del índice de rejuvenecimiento es muy alto $(\mathrm{I} r=0.90)$, lo que indica que la población se encuentra en expansión, como sucede en otras poblaciones de la Sierra de Ayllón (Balaguer Romano et al., 2020). La limitación del acceso al ganado y el desarrollo rápido de los pinares ha tenido un efecto protector sobre los jóvenes tejos. Posteriormente, en el pinar más desarrollado, la abundante fructificación de tejos y de otras especies ornitócoras como Sorbus aucuparia, Ilex aquifolium, Crataegus monogyna o Sambucus nigra han favorecido la dispersión de semillas por túrdidos. De la misma manera, al evaluar las relaciones de proximidad entre los pies tipo 1 y los árboles femeninos más cercanos (posibles progenitoras), midiendo la distancia mínima existente, se determina que la dispersión no tiene relación con la gravedad, lo que parece indicar que el principal factor dispersivo es la zoocoria y que la abundancia de otros frutos carnosos en las proximidades de los tejos favorece la dispersión local de sus semillas. El desplazamiento de la población, que se puede observar en la Fig. 3, sigue una dirección SE dominante, ascendiendo ladera arriba a favor de una menor insolación.

Con este estudio se ha determinado que el tamaño y la densidad de esta población supera la de cualquier otra de la Comunidad de Madrid y del Sistema Central (Fernández González et al., 2015; Balaguer Romano et al., 2020).

En los bordes del área con presencia de tejo (densidades entre 1 y 10 pies/ha, Fig. 2) solo hay ejemplares aislados en una matriz boscosa, por lo que este no transmite carácter a la formación. Sin embargo, en las zonas con densidades entre 10 y 80 pies/ha, el tejo otorga cierto carácter a la formación y entonces podría denominarse "bosque con tejos". Por encima de los 80 pies/ha se puede empezar a considerar la formación como una "tejeda", donde el tejo dificulta el desarrollo de otras especies y modifica claramente las características ecológicas, presentando una composición, estructura y dinámica distintas a la vegetación de las zonas circundantes.

Con el ánimo de promover la conservación de esta excepcional población de tejos proponemos su caracterización como Hábitat 9580*: Bosques mediterráneos de Taxus baccata L. (Serra, 2009) y su inclusión en Listado de Hábitats de la Comunidad de Madrid. 
La Directiva 92/43/CEE de 21 de mayo de 1992 relativa a la conservación de los hábitats naturales define el estado de conservación de un hábitat natural como el conjunto de las influencias que actúan sobre este mismo y sus especies típicas y que pueden afectar a largo plazo a su distribución natural, estructura, funciones, y supervivencia de las especies típicas. Además, el estado de conservación de un hábitat natural se considera favorable cuando:

- Su área de distribución natural sea estable o se amplíe.

- La estructura y las funciones específicas necesarias para su mantenimiento a largo plazo existan y puedan seguir existiendo.

- El estado de conservación de sus especies típicas sea favorable.

Por ello, y para estimar el nivel de calidad y las zonas funcionales específicas del hábitat estudiado, se han valorado la complejidad estructural, la estabilidad-resiliencia, la compacidad y el efecto borde, estableciendo una zonificación de cuatro niveles (Fig. 4).

Destacan dos núcleos principales en cuanto a la presencia, calidad y extensión del hábitat 9580* (subpoblaciones 2 y 4). En otros núcleos este hábitat presenta una calidad intermedia y menor superficie (subpoblaciones 1, 3 y 5) y otras numerosas áreas, con ejemplares más jóvenes, se han definido como de expansión y presencia del tejo, en proceso de adquirir su funcionalidad y estructura características. La superficie de alta calidad del hábitat incluye aprox. 3,31 ha; la de calidad media, 4,63 ha, y el área en expansión, 41,63 ha. El área de presencia de alrededor supone unas 80 ha.

Además, otros criterios se utilizan para valorar la presencia y calidad de un hábitat, como la presencia de especies ca- racterísticas. En este sentido, se identificaron en esta área diecisiete especies características del hábitat 9580* (Lence et al, 2011; García Martí et al, 2018): Aconitum vulparia Rchb., Betula pubescens Ehrh., Corylus avellana L., Erica arborea L., Fagus sylvatica L., Galium rotundifolium L., Hepatica nobilis Miller, Ilex aquifolium L., Melica uniflora Retz., Oxalis acetosella L., Polypodium vulgare L., Salix caprea L., Sorbus aria (L.) Crantz, Sorbus aucuparia L., Taxus baccata L., Vaccinium myrtillus L. y Viola riviniana Rchb.

\section{Conclusiones}

El presente estudio pone de manifiesto la distribución de la población censada en torno a cinco subpoblaciones que, aunque claramente relacionadas, muestran características diferentes; la amplitud ecológica del tejo le ha permitido establecerse en un entorno que le ofrece una variedad de situaciones. Esta plasticidad ya se mostró en otras tejedas del Sistema Central (Fernández González et al., 2015).

La propuesta de caracterización de esta población como Hábitat 9580*: Bosque Mediterráneo de Taxus baccata L., es una importante novedad para el catálogo de hábitats de la Comunidad de Madrid, ya que se trata de un hábitat prioritario no citado hasta el momento. Además, su presencia dentro de un área natural protegida por varias normativas y por estar incluida en la Red Natura 2000, debería encaminar a la administración autonómica a considerar su protección y necesidades de gestión en el correspondiente Plan de Gestión de la ZEC Cuenca del Río Lozoya y Sierra Norte, así como en los planes de gestión de la Reserva de la Biosfera de la Sierra del Rincón y, en el caso de la Comunidad de Castilla-La Mancha, ZEC ES0000164 "Sierra de Ayllón" y al Parque Natural de la Sierra Norte de Guadalajara

\section{- Bibliografía}

- AATT (Asociación Amigos del Tejo y las Tejedas) (2021) Censo y caracterización de la Tejeda del Mosquito. Madrid, $91 \mathrm{pp}$

- Balaguer Romano R., Sainz Ollero H. \& F. Vasco Encuentra (2020) Yew (Taxus baccata L.) population dynamics in the Iberian Mediterranean Mountains: natural regeneration and expansion in East Centra System (Spain). Forest Systems 29 (1): eSC03.

- Fernández González P., Fernández Morcuende A. García Gomáriz E., Rodríguez Rivas M.J., Sánchez Amador E. \& F. Vasco Encuentra (2015). Yew matriarchies of the Sierra de Francia. Dynamics and ecology of recently identified Yew populations in the Central Iberian Mountain Range (Sistema Central). Forest Systems 24 (3): e044.

- García Martí X., Alonso Sánchez A., Fernández González P. \& O. Schwendtner García (2018) Ca- racterización y diagnosis del Hábitat Prioritario "Bosques mediterráneos de Taxus baccata (9580*) de la Cordillera Cantábrica en Castilla y León y País Vasco. LIFE Baccata, inédito.

- IGME (1991) Hoja y Memoria 458 (19-18), Prádena. Mapa Geológico de España, Escala 1.50.000 Madrid. Segunda serie, primera edición, 103 pp.

- IGME (2005) Hoja y Memoria 459 (20-18), Tamajón. Mapa Geológico de España, Escala 1:50.000 Madrid. Segunda serie, primera edición, $163 \mathrm{pp}$

- Lence C., Molina A., Alonso A. \& C. Acedo (2011) Análisis del comportamiento fitosociológico de tejo (Taxus baccata L.) en el noroeste de la Península Ibérica. Spanish Journal of Rural Development Vol. 2, Special Number 2: 7-22.
- Perea, R. (2011) Dispersión y predación de semillas por la fauna: Implicación en la regeneración forestal de bosques templados. Tesis Doctoral. Escuela Técnica Superior de Ingenieros de Montes. Universidad Politécnica de Madrid.

- Rivas Martínez, S. (1987) Mapa de las series de vegetación de España. Ministerio de Agricultura, Pesca y Alimentación. Instituto Nacional para la Conservación de la Naturaleza (ICONA).

- Serra, L. (2009) 9580* Bosques mediterráneos de Taxus baccata. En: Bases ecológicas preliminares para la conservación de los tipos de hábitat de interés comunitario en España (V.VA A.). Ministerio de Medio Amb España.

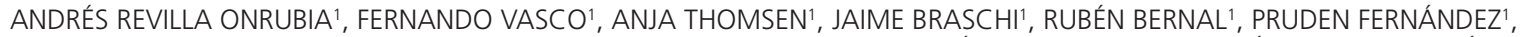
JORGE A. MURILLO', MAR GÉNOVA' ${ }^{1,2}$ / CARTOGRAFÍA: PRUDEN FERNÁNDEZ ${ }^{1}$ 